\title{
Der Referentenentwurf zum Terminservice- und Versorgungsgesetz (TSVG) - Weiterentwicklung der vertragsärztlichen Versorgung
}

\section{Einführung}

Am 24.07.2018 hat das Bundesministerium für Gesundheit (BMG) den Referentenentwurf für das Terminservice- und Versorgungsgesetz (TSVG) vorgelegt, das spätestens zum 01.04.2019 in Kraft treten soll'. Der Referentenentwurf sieht neben zusätzlichen Aufgaben für Terminservicestellen der Kassenärztlichen Vereinigungen (KVen) und die Einführung der elektronischen Patientenakte ab 2021 auch neue Regelungen zu Medizinischen Versorgungszentren (MVZ), zur Bedarfsplanung, insbesondere für das Nachbesetzungsverfahren, sowie zur Erhöhung der Mindestsprechstunden und zur Vergütung vor. Gerade die letztgenannten Themenfelder enthalten Änderungen in der vertragsärztlichen Versorgung, die für radiologische Praxen relevant werden können.

Da ein Gesetzesentwurf nicht von einem einzelnen Bundesministerium in das förmliche Gesetzgebungsverfahren eingebracht werden kann, muss der Referentenentwurf zunächst durch einen Beschluss des Kabinetts der Bundesregierung abgesegnet werden. Eine Kabinettsentscheidung über die Einbringung des Entwurfs des BMG in das Gesetzgebungsverfahren stand bei Redaktionsschluss noch aus. An der vertragsärztlichen Versorgung teilnehmende Radiologen sollten sich dennoch mit den geplanten Änderungen zeitnah befassen. Denn ist der Beschluss über die Einbringung der Gesetzesvorlage gefasst, könnten die geplanten Gesetzesänderungen schon binnen weniger Monate in Kraft treten.

1 Referentenentwurf des Bundesministeriums für Gesundheit vom 23.07.2018, Entwurf eines Gesetzes für schnellere Termine und bessere Versorgung (Terminservice- und Versorgungsgesetz - TSVG), abrufbar unter https:// www.bundesgesundheitsministerium.de/ terminservice-und-versorgungsgesetz.html (letzter Aufruf am 31.08.2018).

\section{Medizinische \\ Versorgungszentren}

Die vertragsärztliche Versorgung durch MVZ stellt einen Schwerpunkt des Referentenentwurfs dar. In einem ersten Schritt sollen bisher bestehende Rechtsunsicherheiten im Zusammenhang mit der Gründung von MVZ sowie der Anstellung von Ärzten durch klarstellende Regelungen ausgeräumt werden. Zudem soll die Position von (durch Ärzte gegründete) MVZ gestärkt werden, um dem Bedürfnis vieler, insbesondere junger Mediziner gerecht zu werden, in einem Angestelltenverhältnis tätig zu sein.

Für Vertragsärzte in gesperrten Planungsbereichen, die auf ihre Zulassung zugunsten einer Anstellung in einem MVZ verzichten, bestanden bisher dahingehend Unsicherheiten, ob sich ihr Vertragsarztsitz in demselben Planungsbereich befinden muss, in dem auch das MVZ seinen Hauptsitz hat. Mit einer Änderung des $§ 103$ Abs. 4a SGB V soll nunmehr klargestellt werden, dass die Anstellung auch in einer Zweigstelle, die in einem anderen Planungsbereich als der Hauptsitz des MVZ gelegen ist, erfolgen kann, wenn der verzichtende Arzt in seinem bisherigen Planungsbereich tätig wird. Das gleiche soll auch für Vertragsärzte bzw. für überörtliche Berufsausübungsgemeinschaften gelten. In der Zulassungspraxis bestand zudem Unklarheit darüber, ob zur Gründung eines MVZ jeweils eine eigene Trägergesellschaft notwendig ist. Laut Begründung des Referentenentwurfs war dies niemals die Intention des Gesetzgebers, sodass nunmehr durch eine Neuformulierung des $\S 95$ Abs. 1a SGB V klargestellt wird, dass eine Trägergesellschaft mehrere MVZ gründen kann.

Bezüglich der Voraussetzungen für die Gründung von MVZ sieht der Referentenentwurf allerdings auch Beschränkungen vor. Erbringer nichtärztlicher Dialyseleistungen nach $\S 126$ Abs. 3 SGB V sollen in Zukunft nur noch zur Gründung „fachbezogener“ MVZ berechtigt sein. Unklar ist aller-

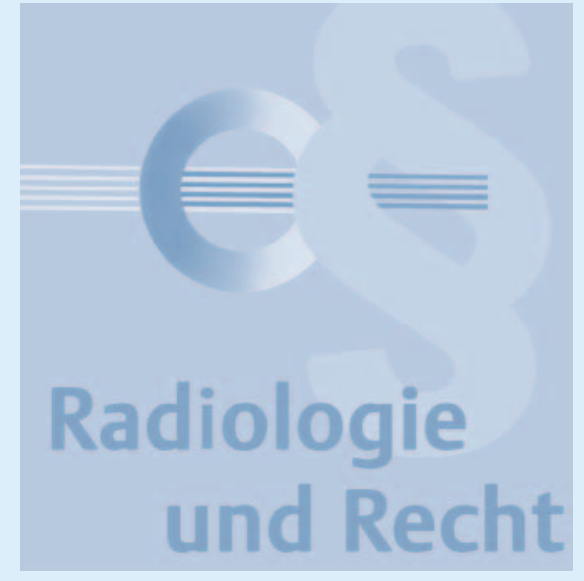

dings, welche Voraussetzungen an die Fachbezogenheit gestellt werden und ob nur Fachärzte für Innere Medizin und Nephrologie ohne oder mit einem Versorgungsauftrag in der Dialyse nach der Anlage 9.1 BMV-Ä und der Qualitätssicherungsvereinbarung zu den Blutreinigungsverfahren in dem MVZ tätig sein dürfen. Mit der Beschränkung verfolgt das BMG das Ziel, die Möglichkeiten für Investoren, die keinen fachlichen medizinischen Bezug aufweisen, an der ambulanten medizinischen Versorgung teilzunehmen, zu beschränken. Bereits seit dem GKV-Versorgungsstrukturgesetz vom 01.01.2012 ist der Gründerkreis von allen Leistungserbringern in $\S 95$ Abs. 1a SGB V auf zugelassene Ärzte, Krankenhäuser, gemeinnützige Träger und Erbringer nichtärztlicher Dialyseleistungen beschränkt worden ${ }^{2}$. Zeitgleich wurde in $\S 95$ Abs. 1a und 6 SGB V eine Bestandsschutzklausel für bis zum 01.01.2012 nach altem Recht zugelassene MVZ eingeführt. Bisher sieht der Referentenentwurf eine solche Bestandsschutzregelung für nach dem 01.01.2012 zugelassene MVZ nicht vor. Da nach § 95 Abs. 6 Satz 3 SGB V einem MVZ die Zulassung zu entziehen ist, wenn die geltenden Gründungsvoraussetzungen länger als 6 Monate nicht mehr vorliegen, hätte die vorgeschlagene Regelung zur Folge, dass den fachfremden MVZ, die von Erbringern nichtärztlicher Dialyseleistungen bereits gegründet und weiterhin betrieben werden, die Zulassung nach 6 Monaten zu entziehen wäre. Dies erscheint verfassungsrechtlich bedenklich.

2 Gesetz zur Verbesserung der Versorgungsstrukturen in der gesetzlichen Krankenversicherung (GKV-VStG) vom 22.12.2011 (BGBI. I, S. 2983). 
Mit diesem Ausschluss werden auf der anderen Seite auch die Möglichkeiten für ärztliche Gründer von MVZ bei der Nachfolgersuche beschränkt. Um diesen Interessen gerecht zu werden, könnte der Gesetzgeber überlegen, statt eines generellen Ausschlusses von Erbringern nichtärztlicher Dialyseleistungen diesen Gründern organisatorische Vorgaben für die Teilnahme an der ambulanten Versorgung zu machen, wie sie sowohl für Vertragsärzte als auch für Krankenhäuser bestehen. Ebenso könnte die Zulassung zum Gründerkreis eines MVZ davon abhängig gemacht werden, dass die mehrheitlichen Anteile eines MVZ von Ärzten gehalten werden. Das Bundessozialgericht (BSG) hat in diesem Zusammenhang erst jüngst auf die Regelung zur Ärztegesellschaft in §23a MBO-Ä verwiesen, die Vorgaben für die ärztliche Tätigkeit in der Form der juristischen Person des Privatrechts enthält ${ }^{3}$.

Dagegen sieht der Entwurf eine Erleichterung hinsichtlich der Übernahme von Gesellschaftsanteilen von ärztlichen Gründern durch im MVZ beschäftigte Ärzte vor. Nach dem geltenden § 95 Abs. 6 Satz 4 SGB V bleibt bei Ärzten, die bei der Gründung eines MVZ auf ihre Zulassung zugunsten der Anstellung verzichten, die Gründereigenschaft bestehen, solange sie in dem MVZ tätig und Gesellschafter des MVZ sind. Wollen ärztliche Gründer aus dem MVZ, z. B. aus Altersgründen, ausscheiden und ihre Gesellschaftsanteile an einen angestellten Arzt, der nicht zum Gründerkreis des MVZ gehört, übertragen, kann es kompliziert werden. Denn nach geltender Rechtslage muss der Übernehmer der Gesellschaftsanteile selbst die Gründungsvoraussetzungen für ein MVZ erfüllen, d. h. er muss zunächst aus seiner Position als angestellter Arzt zur vertragsärztlichen Versorgung zugelassen werden. Um diese Voraussetzungen zu erfüllen, muss die Angestellten-Arztstelle nach $\S 95$ Abs. 9b SGB $V$ zunächst wieder in eine Zulassung umgewandelt werden, sodass der übernehmende Arzt Inhaber der Zulassung werden kann. Nach Übernahme der Gesellschaftsanteile kann der Arzt wiederum zugunsten der Anstellung bei dem MVZ auf seine Zulassung verzichten. Die Handhabung

3 Vgl. BSG, Urteil vom 29.11.2017, Az.: B 6 KA 31/16R. dieses Verfahrens wird von den Zulassungsausschüssen (ZA) bei den Kassenärztlichen Vereinigungen bundesweit unterschiedlich gehandhabt. Einige ZA erkennen die Möglichkeit an, dass die Umwandlung der Arztstelle in eine Zulassung und der Verzicht auf diese zugunsten der Anstellung in derselben Sitzung erfolgen können. Andere ZA verlangen zunächst die Aufnahme und Ausübung der vertragsärztlichen Tätigkeit von bis zu 12 Monaten, bevor ein Arzt wieder auf seine Zulassung zugunsten der Anstellung verzichten kann. Die geplante Neuregelung des §95 Abs. 6 SGB V vereinfacht die Übertragung der Gesellschaftsanteile von angestellten Ärzten mit Gründerstatus enorm. Demnach sollen die Gründungsvoraussetzungen für das $M V Z$ auch dann bestehen bleiben, wenn angestellte Ärzte die Gesellschaftsanteile der Gründer übernehmen, solange sie in dem MVZ tätig sind. Somit können ärztliche Gründer ihre Gesellschaftsanteile an im MVZ angestellte Ärzte übertragen, ohne dass die Übernehmer der Anteile zuvor Inhaber einer vertragsärztlichen Zulassung gewesen sein müssen.

Darüber hinaus soll die Position von MVZ im Verfahren der Nachbesetzung einer vertragsärztlichen Zulassung weiter gestärkt werden. Die ZA sollen in Zukunft dazu verpflichtet werden, im Rahmen der Auswahl der Bewerber die Ergänzung des besonderen Versorgungsangebots des MVZ zu berücksichtigen. Dieses Kriterium können die ZA zwar nach geltendem Recht bereits berücksichtigen, sie sind jedoch bisher nicht dazu verpflichtet, dies in ihre Auswahlentscheidung mit einzubeziehen.

\section{Nachbesetzungsverfahren}

Der Referentenentwurf zum TSVG sieht allerdings auch erhebliche Einschnitte für Nachbesetzungsverfahren in Planungsbereichen mit Zulassungsbeschränkungen vor. Bereits das GKV-VStG von 2012 und das GKV-VSG von 2015 haben hier bereits erhebliche Einschränkungen vorgenommen.

Nach geltender Rechtslage können MVZ und Vertragsärzte die Stelle eines angestellten Arztes nachbesetzen, auch wenn im betroffenen Planungsbereich Zulassungs- beschränkungen angeordnet sind. Dies soll sich nach den Plänen des BMG nunmehr ändern. Die Nachbesetzung einer Arztstelle soll zwar grundsätzlich noch möglich sein, auch wenn Zulassungsbeschränkungen angeordnet sind. Nach den geänderten Regelungen in $\S 103$ Abs. 4a Satz 3 und Abs. 4b Satz 3 SGB V soll der ZA künftig aber die Nachbesetzung einer Arztstelle ablehnen können, wenn diese aus Versorgungsgründen nicht erforderlich ist. Der ZA hat daher ebenso wie bei Anträgen auf Nachbesetzung eines Vertragsarztsitzes zu prüfen, ob ein Bedarf für die Nachbesetzung besteht. Die Nachbesetzung einer AngestelltenArztstelle soll nach der Begründung des Entwurfs damit auf ein „sachgerechtes Maß“ beschränkt werden. Auch wenn das BMG scheinbar eine Gleichstellung der Nachbesetzungsverfahren für Vertragsarztsitze und Arztstellen herbeiführen will, besteht in der geplanten Regelung und dem derzeit geltenden Verfahren zur Nachbesetzung der Vertragsarztsitze der entscheidende Unterschied in der Entschädigung für den Wegfall der Zulassung. Nach § 103 Abs. 3a Satz 13 SGB V hat die KV dem Vertragsarzt oder seinen berechtigten Erben eine Entschädigung zu zahlen, wenn der ZA den Antrag auf Nachbesetzung des Vertragsarztsitzes abgelehnt hat. Eine derartige Entschädigungsregelung im Falle der Ablehnung des Antrags auf Nachbesetzung einer Angestellten-Arztstelle beinhaltet der Referentenentwurf hingegen nicht. Niedergelassene Radiologen, für die derzeit in nahezu allen Planungsgebieten Zulassungsbeschränkungen angeordnet sind, müssten daher bei jedem Ausscheiden eines auf einer Arztstelle angestellten Arztes damit rechnen, dass die Arztstelle entschädigungslos entfällt. Die Regelung ist zu kritisieren, da sie aus bedarfsplanungsrechtlichen Gründen nicht erforderlich ist und zudem den niedergelassenen Ärzten und MVZ keinerlei Planungssicherheit für die Zusammensetzung und den Bestand ihrer Einrichtungen gewährt. Sie kann allenfalls als einseitiges Instrument zum Abbau von Überversorgung angesehen werden, ohne dass jedoch geregelt wird, wie dies im Detail geschehen soll.

Eine Ausnahme sieht der Referentenentwurf jedoch für Nachfolger vor, die zwar zur überversorgten Arztgruppe gehören, aber eine bestimmte Fachrichtung, ein 
Fachgebiet oder eine Schwerpunktkompetenz haben, die innerhalb der Arztgruppe nicht ausreichend vertreten ist. Anträge auf Nachbesetzung mit derartigen Nachfolgern sind stets stattzugeben. Zur Umsetzung dieser Vorgaben soll der Gemeinsame Bundesausschuss (GBA) mit der Einführung einer Neuregelung in $\S 101$ Abs. 1 SGB V die Kompetenz erhalten, die Zusammensetzung der Arztgruppen verbindlich festzulegen, indem er nach Fachrichtungen, Fachgebieten oder Schwerpunktkompetenzen differenzierte Mindest- oder Höchstversorgungsanteile beschließt. Damit sollen Anreize dafür geschaffen werden, dass sich Praxen, die einer überversorgten Arztgruppe angehören, fachlich am Versorgungsbedarf orientieren.

\section{Bedarfsplanung}

Um die Verteilung der Arztsitze stärker am tatsächlichen Versorgungsbedarf zu orientieren, enthält der Referentenentwurf weitere Vorschläge, mit der das BMG die Bedarfsplanung in der ambulanten ärztlichen Versorgung kleinräumiger, bedarfsgerechter und flexibler gestalten will. Der GBA soll dafür die Überprüfung der Bedarfsplanung zügig abschließen und die erforderlichen Anpassungen zum 01.07.2019 regeln. Um in der Zwischenzeit in Bereichen, bei denen laut BMG in besonderem Maße über Versorgungs- und Terminschwierigkeiten geklagt wird, dem Bedarf gerecht zu werden, sollen bis zur Regelung durch den GBA die Zulassungsbeschränkungen für Rheumatologen, Psychiater und Kinderärzte aufgehoben werden. Ärzte dieser Fachrichtungen könnten sich danach innerhalb des Übergangszeitraums ohne Zulassungsbeschränkungen bundesweit um eine vertragsärztliche Zulassung bewerben. Damit der Wegfall der Zulassungsbeschränkungen nicht zu einer Verlegung bereits bestehender Praxen in attraktivere Regionen führt, gilt die Regelung nur für Neuzulassungen und Ärzte, die in den 5 Jahren vor Antragstellung nicht an der vertragsärztlichen Versorgung teilgenommen haben.

Gefördert werden sollen zudem Neuniederlassungen im ländlichen Raum. Dafür ist die Einführung eines neuen $\S 103$ Abs. 3b SGB $\checkmark$ geplant, wodurch die für die Sozialversicherung zuständigen Landesministerien das Recht erhalten, zusätzliche Zulassungen für eine Neuniederlassung in ländlichen Gebieten, für die Zulassungsbeschränkungen angeordnet sind, zu beantragen. Nach dem Wortlaut der geplanten Regelung ist diesem Antrag stets zu entsprechen, sodass die Entscheidung über die Schaffung zusätzlicher Zulassungen allein dem zuständigen Landesministerium obläge.

\section{Erhöhung der Mindestsprechstunden}

Der Referentenentwurf sieht ferner eine Erhöhung der Sprechstunden für gesetzlich versicherte Patienten von derzeit mindestens 20 auf 25 Stunden wöchentlich bei einem vollen Versorgungsauftrag nach § 19a Abs. 1 Ärzte-ZV vor (vgl. § 17 Abs. 1a Satz 1 BMV-Ä). Arztgruppen der grundversorgenden und wohnortnahen Patientenversorgung müssen nach dem Entwurf davon mindestens 5 Stunden wöchentlich als offene Sprechstunden ohne vorherige Terminvereinbarung anbieten. Dazu sollen z. B. Hausärzte, Kinderärzte, konservativ tätige Augenärzte, Frauenärzte, Orthopäden und HNO-Ärzte gehören, wobei die konkrete Regelung, welche Ärzte davon betroffen sind, den Vertragspartnern des Bundesmantelvertrags überlassen werden soll. Für die Überprüfung der Einhaltung der Mindestsprechzeiten soll zudem ein bundesweit einheitliches Verfahren eingeführt werden. Künftig sollen alle KVen die Einhaltung der Versorgungsaufträge insbesondere anhand der abgerechneten Fälle und der im Einheitlichen Bewertungsmaßstab für ärztliche Leistungen (EBM) enthaltenen Gebührenordnungspositionen mit den Angaben zum Zeitaufwand überprüfen.

Bereits nach der geltenden Rechtslage ist nach Ansicht des BSG jedoch zu den mindestens 20 Sprechstunden pro Woche ein Aufschlag von 30 bis $50 \%$ für notwendige Begleitleistungen zu addieren, sodass sich derzeit für den vollen Versorgungsauftrag eine Zeit von insgesamt mindestens 26 bis 30 Arbeitsstunden als Vertragsarzt wöchentlich ermitteln lässt. ${ }^{4}$ Rechnet man den vom BSG geforderten Aufschlag von

4 Vgl. BSG, Urteil vom 13.10.2010, Az.: B 6 KA 40/09 R.
30 bis 50 \% für notwendige Begleitleistungen für den vollen Versorgungsauftrag nach einer Anhebung der wöchentlichen Mindestsprechstundenzeit auf 25 Stunden hinzu, müssten niedergelassene Radiologen zukünftig mindestens 32,5 bis 37,5 Stunden wöchentlich für die Tätigkeit als Vertragsarzt zur Verfügung stehen. Die Möglichkeit der Versorgung von Privatund Krankenhauspatienten wird dadurch zeitlich erheblich eingeschränkt, zumal die Sprechstundenzeiten zu den üblichen Praxiszeiten anzubieten sind.

\section{Sprechstundenvergütung}

Mit finanziellen Anreizen für Ärzte will der Referentenentwurf einen verbesserten Zugang zur haus- und fachärztlichen Versorgung für Patienten erreichen. Durch eine Pauschale bei Neuaufnahme von Patienten soll die Behandlung von Neupatienten extrabudgetär vergütet werden. Als Neupatienten gelten dabei jedoch nicht nur Patienten, die erstmalig einen Termin in der einzelnen Praxis wahrnehmen, sondern auch jene, die wegen einer neu aufgetretenen Krankheit in einer bereits von ihnen aufgesuchten Praxis ärztlich behandelt werden oder in dieser mindestens 1 Jahr nicht vorstellig wurden. Ob diese Regelung für Haus- und Fachärzte gleichermaßen gelten soll, lässt sich dem Referentenentwurf nicht mit Sicherheit entnehmen, da er diesbezüglich nicht kongruent ist. Die vorgeschlagene Gesetzesänderung bezieht sich ihrem Wortlaut nach nur auf die hausärztliche Versorgung. Aus der Begründung ergibt sich hingegen, dass auch Fachärzte für Neupatienten eine Pauschale abrechnen können sollen. Darüber hinaus soll auch die Vermittlung von dringenden Facharztterminen durch den Hausarzt extrabudgetär durch eine bundeseinheitliche Regelung im EBM vergütet werden. Für die Einführung dieser Regelung hat der Bewertungsausschuss bis zum 01.04.2019 Zeit. Verstreicht diese Frist, können die hausärztlichen Leistungserbringer für eine erfolgreiche Vermittlung eines Facharzttermins eine Pauschale in Höhe von 2 Euro abrechnen. Durch die Schaffung finanzieller Anreize für die Terminvermittlung wird im Grunde die Überweisung an einen Facharzt finanziell honoriert. Das ist deshalb bemerkenswert, weil die Überweisung gegen Geld sowohl 
strafrechtlich als auch berufsrechtlich grundsätzlich pönalisiert ist.

\section{Fazit}

Erklärtes Ziel des vom BMG vorgelegten Referentenentwurfs eines Terminserviceund Versorgungsgesetzes (TSVG) ist eine bessere und bedarfsgerechtere Patientenversorgung. Die Auswirkungen der geplanten Änderungen auf die an der vertragsärztlichen Versorgung teilnehmenden Radiologen wären teilweise erheblich. Die freiberufliche Tätigkeit wird insbesondere durch die Regelungen für MVZ, dem Nachbesetzungsverfahren und der Mindestsprechstundenzeiten, wie bereits in den vorhergehenden Gesundheitsreformen, weiter erheblich eingeschränkt. Als Generalargument dienen für alle Regelungen die bestehenden Unterschiede in der
Versorgung. Diese dürfen jedoch nicht dazu missbraucht werden, die ärztliche Tätigkeit über Gebühr zu reglementieren. Zwar sollen einerseits die Übertragung von MVZ-Gesellschaftsanteilen an einen ärztlichen Nachfolger erheblich erleichtert sowie die Möglichkeiten, im ländlichen Bereich eine Zulassung zu erhalten, verbessert werden. Andererseits birgt die geplante Gesetzesänderung hinsichtlich der Nachbesetzung von Arztstellen auch das Risiko eines entschädigungslosen Wegfalls einer Arztstelle bei Ausscheiden des darauf angestellten Arztes. Wegen der zum Teil weitgehenden Einschränkungen für Vertragsärzte und MVZ ist allerdings zu hoffen, dass einige der vorgeschlagenen Regelungen des Entwurfs im Laufe des Gesetzgebungsverfahrens noch nachgebessert bzw. abgeändert werden. Welche der vorgeschlagenen Gesetzesänderungen tatsächlich in Kraft treten werden, bleibt daher bis zum Abschluss des Gesetzgebungsverfahrens abzuwarten. Die erste Verbändeanhörung zum TSVG hat am 22.08.2018 im Bundesministerium für Gesundheit stattgefunden.

Prof. Dr. Peter Wigge

Rechtsanwalt

Fachanwalt für Medizinrecht

Sophia K. Meinecke

Rechtsanwältin

Rechtsanwälte Wigge

Scharnhorststraße 40

48151 Münster

Tel.: (0251) $53595-0$

Fax: (0251) 53595 - 99

Email: kanzlei@ra-wigge.de www.ra-wigge.de 